\title{
CAFEÍNA NÃO ALTERA OS NIVEIS DE IMUNOGLOBULINA A SALIVAR (IGA-S) EM JOGADORES DE VOLEIBOL*
}

\author{
GRAD. JAMILLE LOCATELLI
}

Bacharel e licenciada em educação física - Universidade Federal de Viçosa

Msc. em ciências biológicas - Universidade Federal de Ouro Preto (Minas Gerais - Brasil)

E-mail: jahefi@cedufop.ufop.br

\section{MS. EDMAR LACERDA MENDES}

Mestre em ciência da nutrição - Universidade Federal de Viçosa

Professor assistente, Departamento de Educação Física - Universidade Federal do Triângulo Mineiro (Minas Gerais - Brasil)

E-mail: edpersonal@ig.com.br

\section{GRAD. RAFAEL PIRES DA SILVA}

Bacharel e licenciado em educação física

Msc. em educação física - Universidade Federal de Viçosa (Minas Gerais - Brasil)

E-mail: rafael.pires@ufv.br

\section{DR. SÉRGIO OLIVEIRA DE PAULA}

Doutor em imunologia básica

Professor adjunto, Universidade Federal de Viçosa (Minas Gerais - Brasil)

E-mail: depaula@ufv.br

\section{DR. ANTÔNIO JOSÉ NATALI}

Doutor em fisiologia - Leeds University (Inglaterra)

Professor associado, Universidade Federal de Viçosa (Minas Gerais - Brasil)

E-mail: anatali@ufv.br

\begin{abstract}
RESUMO
Avaliou-se a influência da ingestão de cafeína na resposta da imunoglobulina A salivar (IgA-s) em atletas de voleibol. Usou-se o modelo crossover e duplo-cego. Nove atletas participaram de duas sessões de treino e ingeriram cafeína (6mg/kg de peso corporal) ou placebo. Analisou-se a concentração de IgA-s antes e após as sessões. Os resultados mostraram que não houve diferença significativa $(P>0,05)$ nos níveis de lgA-s pré e pós-treino nos atletas que ingeriram $\operatorname{CAF}(8,54 \pm 4,03 \mathrm{mg} / \mathrm{dL}$ e 7,45 $\pm 4,72 \mathrm{mg} / \mathrm{dL}$, respectivamente), bem como naqueles que tomaram PLA (6,88 $\pm 3,17 \mathrm{mg} / \mathrm{dL}$ e 9,76 $\pm 5,81 \mathrm{mg} / \mathrm{dL}$, respectivamente). Concluiu-se que a suplementação adotada não afetou a resposta da IgA-s durante as sessões de treino realizadas pelos atletas de voleibol.
\end{abstract}

PALAVRAS-CHAVE: Exercício; imunoglobulina A salivar; voleibol; cafeína.

* O presente trabalho não contou com apoio financeiro de nenhuma natureza para sua realização.

Não houve conflitos de interesses para realização do presente estudo. 


\section{INTRODUÇÃO}

A imunoglobulina A salivar (IgA-s) é uma gamaglobulina, proteína plasmática que exibe propriedades imunológicas. A secreção salivar está sob estrito controle do sistema nervoso autônomo. Tanto os nervos simpáticos quanto os parassimpáticos estimulam as glândulas salivares, porém, os efeitos dos nervos parassimpáticos são mais vigorosos e prolongados (EMMELIN, 1987). A estimulação (direta ou por reflexo) do sistema nervoso simpático determina vasoconstrição acentuada, causando redução da produção de saliva e sensação de boca seca (SCHENKELS; VEERMAN; AMERONGEN, 1995).

Exercícios físicos reduzem os níveis da imunoglobulina A ( $\lg A)$ nos fluidos nasal e oral (PACQUE et al., 2007). O primeiro estudo a demonstrar modificações nos níveis de IgA em resposta ao exercício físico foi publicado na literatura médica em 1982 por Tomasi et al. (TomAsl et al., 1982). Esse estudo reportou que homens e mulheres de uma equipe de esqui exibiram menor concentração de IgA-s em repouso, quando comparados ao grupo controle da mesma idade. Desde então, um crescente número de investigações sobre as respostas da IgA ao exercício em atletas de diferentes modalidades, tanto de longa quanto de curta duração, têm sido divulgados - isto é, vela (NeVILle; GleEson; Folland, 2008), corredores de ultrarresistência (AltimarI et al. 2006), jogadores de futebol (SARI-SARRAF et al., 2008), jogadores de rugby (KocH et al., 2007) e ciclistas (LAING et al., 2005). Todavia, alterações na concentração da IgA-s em resposta ao exercício físico parecem ocorrer em função de vários fatores, incluindo a duração e a intensidade do exercício, o período de treinamento que precede o exercício e o estresse psicológico associado ao treinamento e à competição (GleEson, 2007; Fricker et al., 1999; Mackinnon; Hooper, 1994).

Algumas estratégias nutricionais com foco no rendimento esportivo ganham campo nas investigações científicas que envolvem atletas de elite. Diversos estudos demonstram que a ingestão de pequenas doses de cafeína (3-6 mg/kg de peso corporal) é capaz de otimizar o desempenho atlético em diferentes tipos de exercício, sobretudo em atividades de longa duração (Altimarl et al., 2006; DOHERTY; SMITH, 2005). O efeito da cafeína no desempenho durante a prática de exercícios físicos se deve, provavelmente, à sua ação ergogênica ao alterar a percepção do esforço, aumentar o estado de alerta e aumentar a duração do exercício até a fadiga (DOHERTY; Smith, 2005; Paluska, 2003; James; Wilson; Askew, 2004; Kalmar; Cafarelli, 2004). Acredita-se, ainda, que a ação estimulante da cafeína no SNC envolva a estimulação do sistema nervoso simpático, aumentando a liberação e, consequentemente, a ação das catecolaminas, que, por sua vez, levam a taquicardia, sudorese, dilatação dos brônquios e pupilas (Altimarl et al., 2006). 
As células do sistema imunológico possuem receptores para $\beta$-endorfinas, catecolaminas, cortisol e diversos outros mediadores envolvidos na reação ao estresse (Pedersen; SteensBerg, 2002). O exercício físico intenso aumenta os níveis plasmáticos de diversos hormônios estressores que influenciam a resposta imune (MoYNIHAN, 1998). Considerando que tanto o exercício quanto a ingestão de cafeína podem estimular o sistema simpático, especula-se que a ingestão de cafeína associada ao estresse fisiológico decorrente do treinamento físico poderia afetar a mobilização de IgA-s em atletas.

Nesse sentido, o presente estudo teve como objetivo avaliar a influência da ingestão de cafeína na resposta da IgA-s em atletas de voleibol durante uma sessão de treino.

\section{MATERIAIS E MÉTODOS}

\section{AMOSTRA E DESENHO EXPERIMENTAL}

Participaram deste estudo nove atletas masculinos com idade de 22,7 $\pm 3,0$ anos (média \pm desvio padrão), peso corporal de 80,86 \pm 8,6I kg e IMC de 24,0 $\pm 2,5 \mathrm{~kg} / \mathrm{m}^{2}$, filiados à Associação Atlética Acadêmica da Universidade Federal de Viçosa (AAA-UFV). Os atletas foram selecionados seguindo os seguintes critérios de inclusão: grau de experiência prévia da modalidade ( $\geq 2$ anos) e que participavam regularmente do treinamento ( 23 vezes por semana). Este estudo foi previamente aprovado pelo Comitê de Ética em Pesquisas com Seres Humanos da Universidade Federal de Viçosa e os sujeitos assinaram o termo de consentimento livre e esclarecido.

Os sujeitos foram submetidos a duas sessões de treino de voleibol, com I5 dias de intervalo entre elas. Nesse período entre as avaliações, os atletas seguiram o treinamento habitual. Na primeira sessão, cinco atletas foram escolhidos aleatoriamente para consumirem solução contendo cafeína (CAF). Os demais atletas consumiram solução placebo (PLA). Os tratamentos foram invertidos na segunda sessão. Adotou-se um desenho cruzado, balanceado (crossover) e duplo-cego, de maneira que um grupo de cinco avaliados iniciasse o teste com ingestão de bebida distinta. Cabe destacar que o estudo impõe nos dois procedimentos de suplementação com cafeína um desenho intraindivíduos, no qual os mesmos indivíduos foram considerados como controle e experimentais. 
Cada sessão de treino teve duração de 60 minutos, sendo 15 minutos de aquecimento e alongamento, 15 minutos de fundamentos técnicos e 30 minutos de coletivo. Os exercícios para aquecimento e alongamento foram compostos por: movimentos de lançamento de bola, saltos, corridas com mudanças de direção e alongamentos de membros superiores, tronco e membros inferiores. $\bigcirc$ treinamento técnico foi composto por: movimentações de bloqueio, ataque e defesa.

\section{HÁBITOS ALIMENTARES}

Após avaliação dos hábitos alimentares dos atletas, por meio de questionário e de recordatório alimentar de 24 horas (anamnese alimentar), foi determinado o tempo de jejum de cafeína para cada atleta. Os indivíduos que consumiam em média $12 \mathrm{mg} / \mathrm{Kg}$ de peso de cafeína por dia (consumo de produtos, como, por exemplo, chocolate, café, refrigerantes, dentre outros) em sua dieta rotineira tiveram o consumo do ergogênico suspenso por 72 horas, antes das sessões de treino, para evitar que os efeitos de estimulação simpática da cafeína fossem mascarados. Os demais indivíduos abstiveram-se do consumo de cafeína por 24 horas.

\section{INGESTÃO DE CAFEÍNA}

A massa corporal dos atletas foi aferida antes das sessões de treino para determinação da quantidade de cafeína a ser ingerida por cada indivíduo. A cafeína foi misturada a uma bebida (suco) que foi preparada para cada atleta, de acordo com o peso corporal (6 ml/kg de peso corporal). Ambas as bebidas (PLA e CAF) tinham cor, sabor e textura semelhantes. Cada indivíduo ingeriu 6 mg de cafeína por kg de peso corporal, imediatamente I hora antes da sessão de treino. Essa dosagem foi determinada com base em estudo anterior (BISHOP et al., 2006).

\section{COLETA DE SALIVA E DOSAGEM DE IGA-S}

Foi coletado I ml de saliva em repouso, antes da ingestão da bebida e I ml imediatamente após a sessão de treino. A saliva foi coletada em eppendorfs e armazenada em freezer a temperatura entre $2^{\circ} \mathrm{C}$ e $8^{\circ} \mathrm{C}$ para análise.

A dosagem de IgA-s foi realizada por "imunonefelometria", conforme instruções do fabricante do kit " $\mathrm{N}$ anti-soros contra a imunoglobulina IgA" (Dade Behring, Marburg, Alemanha). Para tanto, as amostras de saliva foram diluídas com N diluente a I:20 e aplicadas no sistema BN* I/BN ProSpec que executou todos os passos do protocolo automaticamente, emitindo feixes de luz que correspondem à concentração de $\lg \mathrm{A}$. 
Foi aplicado o teste de normalidade, e foi realizada posterior análise descritiva dos dados. Os valores apresentados no texto correspondem à média \pm desvio padrão. As comparações dos níveis de IgA-s considerando as situações PLA e CAF, antes e depois do esforço, foram feitas utilizando o tratamento estatístico ANOVA two-way. Adotou-se o nível de significância de até 5\%. A análise estatística foi realizada usando o software SigmaStat, versão 3.0.

\section{RESULTADOS}

Quando os atletas ingeriram PLA, a concentração de IgA-s após o treino aumentou ligeiramente $(6,88 \pm 3,17 \mathrm{mg} / \mathrm{dl})$ em relação aos valores de IgA-s prétreino $(9,76 \pm 5,8 \mathrm{l} \mathrm{mg} / \mathrm{dl})$, mas a diferença não foi estatisticamente significante $(P<$ 0,05; Gráfico I). Quando os atletas consumiram CAF, os valores médios dos níveis de lgA-s após a sessão de treino $(8,54 \pm 4,03 \mathrm{mg} / \mathrm{dl})$ tenderam a uma diminuição em relação aos valores pré-treino (7,45 \pm 4,72 mg/dl); entretanto, não houve significância estatística $(P<0,05 ;$ Gráfico I).

Não houve diferença estatística também entre a variação $(\Delta)$ média nos níveis de IgA-s pré e pós-treino nos dois procedimentos experimentais $(\Delta=2,88 \pm 4$, $\mathrm{PLA} ; \Delta=-1,09 \pm 5,49, \mathrm{CAF}$ ). Os níveis de IgA-s pré-treino não foram diferentes entre as duas sessões de treino.

Gráfico I: Concentração de lgA-s (mg/dL) pré e pós-treino dos atletas de voleibol quando ingeriram placebo e cafeína

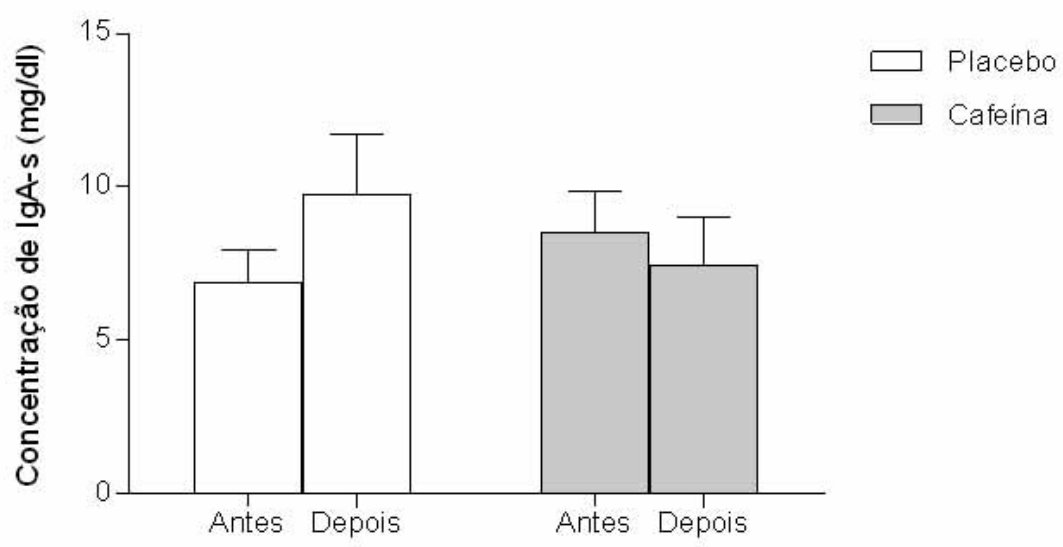




\section{DISCUSSÃO}

O presente estudo teve como objetivo avaliar a resposta da IgA-s em uma sessão de treino de voleibol após a ingestão de cafeína I hora antes do treino. A literatura científica pertinente é vasta em publicações referentes à ação do exercício sobre a resposta da IgA (SILVA et al., 2009; SAYGIN et al., 2006). Contudo, é conhecido apenas um estudo que analisou a associação entre a ingestão prévia de cafeína ao exercício e subsequentes níveis de IgA-s. Nesse estudo, Bishop et al (2006) investigaram I I ciclistas treinados pedalando por 90 minutos a $70 \%$ do $\mathrm{VO}_{2}$ de pico uma hora após a ingestão de $6 \mathrm{mg} / \mathrm{kg}$ de peso corporal e observaram uma elevação marcada das concentrações de IgA-s tanto durante como imediatamente após o exercício. Os resultados do presente estudo, entretanto, sugerem que a ingestão de cafeína por atletas de voleibol I hora antes de uma sessão de treino não está associada a alterações nas concentrações absolutas de lgA-s após o treinamento. As razões para a discrepância entre os resultados dos dois estudos não é clara, mas pode estar parcialmente relacionada com a duração, intensidade, tipo de treino e modalidade.

O exercício é um potente modulador do sistema nervoso simpático, exercendo influência na liberação de catecolaminas. Seria plausível supor, portanto, que a superposição da ingestão de cafeína ao estresse do exercício aumentasse a secreção de IgA-s em atletas de voleibol durante uma sessão de treino. No entanto, não foi o que ocorreu. Sabe-se que as concentrações de lgA-s são reduzidas tanto durante (GLEESON et al., 1999) quanto após (NIEMAN et al., 2002) períodos de exercício extenuantes tanto pela intensidade como pela duração. Essa redução parece estar associada ao aumento nos níveis de cortisol, que também é elevado em exercícios agudos vigorosos seguidos da ingestão de cafeína (HuCKLEBRIDGE, CLow; Evans, 1998; LAURENT et al., 2000). Lovallo et al. (2006) afirmam que a cafeína eleva a secreção de cortisol, assim como o exercício, e ressalta que, quando combinamos os dois fatores, há um efeito substancial e prolongado em homens. Para tanto, no presente estudo observou-se uma tendência a um ligeiro aumento nos níveis de IgA-s no PLA, o que pode estar relacionado a uma expressividade reduzida do cortisol pela ausência da cafeína nesse grupo.

Além disso, analisando as mais diversas formas de exercício físico, principalmente os esportes acíclicos com bola, o que se observa é um prolongado período em exercício, porém, com intensidades variadas. A maioria dos esportes, como tênis, basquetebol, voleibol, entre outros, caracteriza-se por momentos de intensidade alta, intercalados com períodos de intensidade moderada ou rápido repouso. A duração (60 minutos) e o tipo de estímulo do exercício aplicado no 
presente estudo podem ter sido insuficientes para assinalar respostas hormonais consideráveis que viessem a promover alterações nas concentrações de IgA-s. Além disso, pela dificuldade prática de se avaliar diretamente o esforço em atividades acíclicas, o efeito de exercícios intermitentes sobre a IgA-s não está bem esclarecido e carece de mais investigações. Seria interessante que estudos futuros mensurassem o nível de condicionamento dos atletas, assim como os indicadores de respostas metabólicas ao exercício.

Os valores $\Delta$ de variação indicam uma grande variabilidade nos níveis de IgA-s entre os atletas. De fato, aspectos individuais como a idade, o gênero, o nível de condicionamento, fatores nutricionais, entre outros, exercem influência sobre as concentrações de IgA; a variação dos níveis de IgA-s entre os sujeitos é amplamente observada (SILVA et al., 2009). Saygin et al. (2006) também encontraram diferenças nos valores basais de lgA-s entre jogadores de voleibol, corredores de longa distância e indivíduos sedentários. Os jogadores de voleibol apresentaram níveis mais elevados do que sedentários e corredores, enquanto estes exibiram níveis maiores do que aqueles. As razões para essa maior variabilidade entre os atletas de elite assim como as demais influências dessa variação biológica necessitam de mais investigações.

Seria possível que com uma dose de cafeína acima dos valores administrados no presente estudo pudesse haver alguns efeitos positivos sobre a IgA-s, entretanto, seria extremamente desaconselhável. Após o consumo da bebida contendo cafeína, alguns atletas relataram sintomas de inquietação e estimulação simpática exacerbada, como insônia, tremor e taquicardia, de modo que doses mais elevadas poderiam afetar sobremaneira o sistema nervoso central, gerando efeitos adversos em indivíduos mais sensíveis à cafeína. Além disso, os efeitos ergogênicos da cafeína têm sido observados em pesquisas que utilizaram metade da dose administrada neste estudo (GraHAM, 200I), não justificando, portanto, seu consumo excessivo.

\section{CONCLUSÃO}

Os resultados do presente estudo sugerem que o consumo de cafeína (6 mg/ kg de peso corporal) não afeta a resposta da lgA-s durante uma sessão de treino em atletas masculinos de voleibol. Como o controle da IgA-s em atletas é extremamente relevante e viável, principalmente por se tratar de método prático e não invasivo, estudos futuros podem ajudar a elucidar os mecanismos biológicos que regulam ou modulam as alterações na IgA em relação à cafeína decorrentes de outros modelos de exercício e ações experimentais. 


\section{Caffeine does not change Salivary Immunoglobulin A (s-lgA) levels in volleyball players}

ABSTRACT: We tested the influence of caffeine (CAF) ingestion on the salivary immunoglobulin A (s-lgA) of volleyball's athletes. In a cross-over and double blind way, nine male volleyball players performed two training sessions within 15 days interval when ingested either CAF (6 mg/kg b.w.) or placebo (PLA) one hour before. The levels of s-lgA were measured at rest and after exercise. The results showed that the levels of S-IgA at rest were not statistically different $(P>0.05)$ from those after exercise when athletes ingested either CAF (8.54 \pm $4.03 \mathrm{mg} / \mathrm{dL}$ and $7.45 \pm 4.72 \mathrm{mg} / \mathrm{dL}$, respectively) or PLA (6.88 $\pm 3.17 \mathrm{mg} / \mathrm{dL}$ and 9.76 $\pm 5.81 \mathrm{mg} / \mathrm{dL}$, respectively). It is concluded that caffeine ingestion did not affect the s-lgA response during the training session performed by the athletes.

KEY WORDS: Exercise; salivary immunoglobulin A; volleyball; caffeine.

\section{Cafeína no altera los niveles de imunoglobulina salivar, en jugadores de voleibol}

RESUMEN: Se avalio la influencia de la ingestión de cafeína en la respuesta de a inmunoglobulina A salivar (IgA-s) en atletas de voleibol se uso el modelo crossover y doble-cego. Nueve atletas participaran de dos sesiones de entrenamiento y ingirieron cafeína (6 mg de peso corporal) o placebo. Se analizo la concentración de lgA-s antes y después de la sesión. Los resultados mostraron que no hubo diferencia significativa $(P>0,05)$ en los niveles de (IgA-s) pre e pos entrenamiento en los atetas que ingirieron CAF $(8,54 \pm 4,03$ $\mathrm{mg} / \mathrm{dL}$ e 7,45 $\pm 4,72 \mathrm{mg} / \mathrm{dL}$, respectivamente) como en aquellos que tomaron PLA (6,88 $\pm 3,17 \mathrm{mg} / \mathrm{dL}$ e $9,76 \pm 5,81 \mathrm{mg} / \mathrm{dL}$, respectivamente). Se concluyo que la suplementación adoptada no afecto la respuesta de la lgA-s durante las sesiones del entrenamiento realizados por los atletas de voleibol.

PALABRAS CLAVES: Ejercicio; imunoglobulina A salivar; voleibol; cafeína.

\section{REFERÊNCIAS}

ALTIMARI, L. R; MORAES, A. C; TIRAPEGUI, J; MOREAU, R. L. M. Cafeína e performance em exercícios anaeróbios. Revista Brasileira de Ciências Farmacêuticas, São Paulo, v. 42, p. 17-27, 2006.

BISHOP, N. C; WALKER, G. J.; SCANLON, G. A.; RICHARDS, S.; ROGERS, E. Salivary IgA responses to prolonged intensive exercise following caffeine ingestion. Med. Sci. Sports Exerc., Madison, v. 38, p. 513-9, 2006. 
DOHERTY, M.; SMITH, P. M. Effects of caffeine ingestion on rating of perceived exertion during and after exercise: a meta-analysis. Scand. J. Med. Sci. Sports, Copenhagem, v. I5, p. 69-78, 2005.

EMMELIN, N. Nerve interaction in salivary glands. J. Dent. Res., Washington, v. 66, n. 2, p. 509-517, 1987.

FRICKER, P. A.; MCDONALD, W. A.; GLEESON, M.; CLANCY, R. L. Exercise-associated hypogammaglobulinemia. Clin. J. Sport Med., New York, v. 9, p. 46-8, 1999.

GLEESON, M.; MCDONALD, W. A.; PYNE, D. B.; CRIPPS, A. W.; FRANCIS, J. L.; FRICKER, P. A. et al. Salivary IgA levels and infection risk in elite swimmers. Med. Sci. Sports Exerc., Madison, v. 31 , p. 67-73, 1999.

GLEESON, M. Immune function in sport and exercise. J. Appl. Physiol., Washington, v. 103, p. 693-9, 2007.

GRAHAM, T. E. Caffeine, coffee and ephedrine: Impact on exercise performance and metabolism. Can. J. Appl. Physiol., Champaign, v. 26 p. SI 19-S203, 2001 . Suppl.

HUCKLEBRIDGE, F; CLOW, A.; EVANS, P. The relationship between salivary secretory immunoglobulin A and cortisol: neuroendocrine response to awakening and the diurnal cycle. Int. J. Psychophysiol., Amsterdam, v. 31, p. 69-76, 1998.

JAMES, R. S.; WILSON, R. S., ASKEW, G. N. Effects of caffeine on mouse skeletal muscle power output during recovery from fatigue. J Appl Physiol, Washington, v. 96, p. 545-52, 2004.

KALMAR, J.M.; CAFARELLI, E. Central fatigue and transcranial magnetic stimulation: effect of caffeine and the confound of peripheral transmission failure. J. Neurosci. Methods, Amsterdam, v. 138, p. 15-26, 2004.

KOCH, A. J.; WHERRY, A. D.; PETERSEN, M. C.; JOHNSON, J. C.; STUART, M. K.; SEXTON, W. L. Salivary immunoglobulin A response to a collegiate rugby game. J. Strength Cond. Res., Lincoln, v. 21 , p. 86-90, 2007.

LAING, S. J.; GWYNNE, D.; BLACKWELL, J.; WILLIAMS, M.; WALTERS, R.; WALSH, N. P. Salivary IgA response to prolonged exercise in a hot environment in trained cyclists. Eur. J. Appl. Physiol., Berlin, v. 93, p. 665-7I, 2005.

LAURENT, D.; SCHNEIDER, K. E.; PRUSACYZK, W. K.; FRANKLIN, C.; VOGEL, S. M.; KRSSAK, M.; PETERSEN, K. F.; GOFORTH, H. W.; SHULMAN, G. I. Effects of caffeine on muscle glycogen utilization and neuroendocrine axis during exercise. J. Clin. Endocrinol. Metab., Philadelphia, v. 85, p. 2170-2175, 2000. 
LOVALLO, W. R.; FARAG, N. H.; VINCENT, A. S.; THOMAS, T. L.; WILSON, M. F.; Cortisol responses to mental stress, exercise, and meals following caffeine intake in men and women. Pharmaco.I Biochem. Behav., Phoenix, v. 83, p. 44I-447, 2006.

MACKINNON, L. T.; HOOPER, S. Mucosal (secretory) immune system responses to exercise of varying intensity and during overtraining. Int. J. Sports Med., Stuttgart, v. I 5, p. S179-83, 1994. Suppl 3.

MOYNIHAN, J. A.; CALLAHAN, T. A.; KELLEY, S. P.; CAMPBELL, L. M. Adrenal hormone modulation of type I and type 2 cytokine production by spleen cells: dexamethasone and dehydroepiandrosterone suppress interleukin-2, interleukin-4, and interferon-gamma production in vitro. Cell Immunol., New York, v. I84, p. 58-64, 1998.

NEVILLE, V.; GLEESON, M.; FOLLAND, J. P. Salivary IgA as a risk factor for upper respiratory infections in elite professional athletes. Med. Sci. Sports Exerc., Madison, v. 40, p. I228-1236, 2008.

NIEMAN, D. C.; HENSON, D. A.; FAGOAGA, O. R.; UTTER, A. C.; VINCI, D. M.; DAVIS, J. M. Change in salivary IgA following a competitive marathon race. Int. J. Sports Med., Stuttgart, v. 23, p. 69-75, 2002.

PACQUE, P. F.; BOOTH, C. K.; BALL, M. J.; DWYER, D. B. The effect of an ultra-endurance running race on mucosal and humoral immune function. J. Sports Med. Phys. Fitness, Torino, v. 47, p. 496-501, 2007.

PALUSKA, S. A. Caffeine and exercise. Curr. Sports Med. Rep., Philadelphia, v. 2, p. 213-219, 2003.

PEDERSEN, B.K.; STEENSBERG, A. Exercise and hypoxia: effects on leukocytes and interleukin6-shared mechanisms? Med. Sci. Sports Exerc., Madison, v. 34, p. 2004-13, 2002.

SAYGIN, O.; KARACABEY, K.; OZMERDIVENLI, R.; ZORBA, E.; ILHAN, F.; BULUT, V. Effect of chronic exercise on immunoglobin, complement and leukocyte types in volleyball players and athletes. Neuro Endocrinol. Lett., Weinheim, v. 27, p. 27I-6, 2006.

SARI-SARRAF, V.; REILLY, T.; DORAN, D.; ATKINSON, G. Effects of repeated bouts of soccer-specific intermittent exercise on salivary lgA. Int. J. Sports Med., Stuttgart, v. 29, p. 366-37I, 2008.

SCHENKELS, L. C. P. M.; VEERMAN, E. C. I.; AMERONGEN, A. V. N. Biochemical Composition of human saliva in relation to other mucosal fluids. Crit. Rev. Oral. Biol. Med., Boca Raton, v. 6, n. 2, p. 161-175, 1995. 
SILVA, R. P.; NATALI, A. J.; DE PAULA, S. O.; LOCATELLI, J.; MARINS, J. C. B. Imunoglobulina A salivar (IgA-s) e exercício: relevância do controle em atletas e implicações metodológicas. Rev. Bras. Med. Esporte, São Paulo, v. 15, n. 6, p. 459-466, 2009.

TOMASI, T. B.; TRUDEAU, F. B.; CZERWINSKI, D.; ERREDGE, S. Immune parameters in athletes before and after strenuous exercise. J. Clin. Immunol, New York, v. 2, p. I73- I78, 1982.

Recebido: 26 out. 2008

Aprovado: 08 fev. 2010

Endereço para correspondência Jamille Locatelli Universidade Federal de Ouro Preto - Centro Desportivo Campus Universitário, Morro do Cruzeiro Ouro Preto-MG CEP 35400-000 